\title{
La implementación local de la atención primaria en la Argentina: estudio de caso en el municipio de Lanús, provincia de Buenos Aires
}

\author{
Local implementation of the primary health care in Argentina: case study in Lanús \\ municipality, Buenos Aires province
}

Sara Ardila Gómez , Pierre de Paepe ${ }^{2}$, Leandro Luciani Conde³ ${ }^{3}$ Alicia Stolkiner ${ }^{4}$

\begin{abstract}
'Becaria Doctorado en el Consejo Nacional de Investigaciones Científicas y Técnicas (Conicet), Departamento de Salud Comunitaria, Universidad Nacional de Lanús; Docente e Investigadora de Cátedra II Salud Pública/Salud Mental en la Facultad de Psicología, Universidad de Buenos Aires - Buenos Aires (BA), Argentina. saraardi@gmail.com
\end{abstract}

${ }^{2}$ Doctor of Medicine, Master of Public Health; Investigador del Departamento de Salud Pública, Instituto de Medicina Tropical - Amberes, Bélgica.

pdpaepe@itg.be

${ }^{3}$ Doctorado en Ciencias Sociales pela Universidad de Buenos Aires (UBA) Buenos Aires (BA); Coordinador del Departamento de Salud Comunitaria: Docente e Investigador en la Universidad Nacional de Lanús - Lanús (BA); Profesor Adjunto e Investigador de Cátedra II Salud Pública/Salud Mental en la Facultad de Psicología, Universidad de Buenos Aires - Buenos Aires (BA), Argentina.

Ilucianiconde@hotmail.com

4Profesora Titular e Investigadora en el Departamento de Salud Comunitaria, Universidad Nacional de Lanús - Lanús (BA), Argentina. Profesora Titular e Investigadora, Cátedra II Salud Pública/Salud Mental, Universidad de Buenos Aires; Coordinadora del Nodo Argentino de la Red de Investigación en Sistemas y Servicios de Salud del Cono Sur - Buenos Aires (BA), Argentina.

astokiner@fibertel.com.ar
RESUMEN Se presentan resultados en Argentina de la segunda etapa de un estudio multicéntrico sobre Atención Primaria en Salud, realizado por la Universidad Nacional de Lanús y por la Red de Investigación en Sistemas y Servicios de Salud del Cono Sur, financiado por el International Development Research Center (Canadá). Se indagó sobre la traducción en servicios, prácticas y procesos de los abordajes y modelos de la Atención Primaria en Salud. Eso es un estudio de caso que aplicó el cuestionario de Evaluación Rápida de Atención Primaria en Salud (Barbara Starfield; adaptación y validación por Almeida/Macinko) a usuarios y profesionales de centros de salud del Municipio de Lanús. Se entrevistó a gestores y fueron realizados talleres participativos. Los resultados señalan que el índice global de desempeño de la Atención Primaria en Salud en el municipio fue satisfactorio, con puntuaciones altas en las dimensiones 'vínculo con profesionales' $y$ 'formación profesional', y bajas en 'acceso'y 'orientación a la comunidad'.

PALABRAS CLAVE: Atención Primaria de Salud; Primer Nivel de Atención; Desempeño; Argentina.

\begin{abstract}
Results of the second phase of a multicenter study on Primary Health Care are here presented, which were implemented by the National University of Lanús and by the Network of Research on Health Systems and Services of the Southern Cone, financed by the International Development Research Center (Canada). The objective of this phase was to describe the translation of Primary Health Care models into practices, services, and processes. It is a case study that applied the survey of Primary Health Care Fast Evaluation (Barbara Starfield, adaptation by Almeida/Macinko) to users and staff of health centers at Lanús municipality. Primary Health Care managers were interviewed, and participatory workshops were organized. Results indicate that the global index of performance of the Primary Health Care in the municipality was satisfactory with high scores on the dimensions 'professional link' and 'professional training,' and low on 'access' and on 'community guidance'.
\end{abstract}

KEYWORDS: Primary Health Care; First Level of Care; Performance; Argentina. 


\section{Introducción}

Desde su promulgación, se ha planteado que la Atención Primaria de la Salud (APS) constituye una herramienta propicia para la mejora de las condiciones de salud de la población, y, en un sentido amplio, para la materialización del derecho a la salud. No obstante, las formas en que ésta se ha llevado a la práctica han variado entre los países, e incluso al interior de los países mismos, y ha conducido a resultados disímiles. A partir de este marco, en el 2009 la Red de Investigación en Sistemas y Servicios de Salud comenzó el "Estudio multicéntrico en APS: modelos asistenciales, integración al sistema de salud e intersectorialidad en contextos urbanos en Argentina, Brasil, Paraguay y Uruguay", con financiación del International Development Research Center/Canadian Institute of Interfaith Dialog (IDRC/CIID) de Canadá. Se trataba de realizar estudios en cada uno de los países con herramientas similares que permitieron la comparación. La sede en la Argentina fue la Universidad Nacional de Lanús, en el conurbano bonaerense.

El objetivo general de la investigación fue

identificar limites y posibilidades para reorientar la APS como estrategia para alcanzar sistemas de salud integrados y universales, considerando el grado de segmentación del sistema de salud y la fragmentación en la provisión de los servicios. (RED DE INVESTIGACIÓN EN SISTEMAS Y SERVICIOS DE SALUD DEL CONO SUR, 2011).

El estudio tuvo dos etapas: un análisis panorámico de la APS en los cuatro países - Argentina, Brasil, Paraguay y Uruguay - realizado en 2010, y estudios de casos en centros urbanos en 2011. En este trabajo se exponen resultados de esta segunda etapa en la Argentina, realizada en el municipio de Lanús.

Es necesario señalar que esta investigación se enmarca en el campo de la investigación en sistemas y servicios de salud (ALMEIDA, 2000), para lo cual resultó significativo que se realizara en el municipio en el que está la universidad, que fue su sede. Esa una universidad estatal nacional tiene como misión primaria contribuir a través de la producción y distribución de conocimiento y de innovaciones cientifico-tecnológicas, al desarrollo económico, social y cultural de la región, a fin de mejorar su calidad de vida y fortalecer los valores democráticos en el conjunto de la sociedad. (UNIVERSIDAD NACIONAL DE LANÚS, 2010).

La actividad del equipo de investigación se incorporó en el marco de acuerdos establecidos de cooperación con el municipio y retomó los de otro equipo de investigación de la universidad que realizó un estudio sobre desigualdades en salud en ese municipio (ALAZRAQUI; SPINELLI, 2008).

\section{Contexto}

\section{La Atención Primaria de Salud en la Argentina}

Como se señalara, en la primera etapa del estudio se analizó el desarrollo de la APS en cada país a escala nacional. Para ello, se diseñó una guía de relevamiento de indicadores socioeconómicos y sanitarios y una matriz de análisis para los datos, cuyas dimensiones fueron: capacidad de conducción, financiación, recursos (provisión y recursos humanos), integralidad y continuidad de cuidado y articulación intersectorial/relaciones de la APS con actores sociales y políticos. La segmentación del sistema y la gobernanza (BURSZTYN et al., 2010) fueron categorías analíticas transversales a las dimensiones estudiadas. Los resultados sobre Argentina fueron presentados en otro trabajo (STOLKINER; COMES; GARBUS, 2011), del cual es necesario retomar algunos elementos a fin de contextualizar la situación en el municipio de estudio de esta segunda etapa.

Para empezar, cabe resaltar que el período temporal del estudio coincide en la Argentina con el posterior a la crisis económica, social y política del 2001, durante el cual hubo modificaciones importantes de las políticas sociales en el marco de una etapa de crecimiento económico sostenido. No obstante, los cambios en el sistema de salud no fueron tan notables como en otras áreas, por ejemplo, el sistema previsional. No hubo una reforma estructural del sistema de salud que comprende 
tres subsectores: el estatal, cuya financiación fundamental proviene de recursos del presupuesto de la Nación, provincias y/o municipios; el de obras sociales que se financia con el aporte de los trabajadores y empleadores; y el privado con financiamiento de los usuarios.

El sistema se ha caracterizado por la segmentación, fragmentación y heterogeneidad. Estas se manifiestan en la coexistencia de múltiples instituciones, tanto en lo financiero como en la provisión de servicios, sin formas de coordinación que faciliten una distribución adecuada de los diferentes niveles de atención y eviten superposiciones o falta de disponibilidad. La heterogeneidad define la existencia de normas particulares para cada organización, las cuales suponen diferencias en la captación de recursos, en las formas de utilización, y en los derechos reconocidos a la población a cargo (BELMARTINO, 2005).

Por otro lado, si bien la APS está planteada como estrategia y meta en los documentos oficiales, en las prácticas se la considera equivalente al primer nivel de atención. Así mismo, su desarrollo es heterogéneo debido a que las prestaciones del primer nivel, fundamentalmente sostenidas en el subsector estatal, dependen mayoritariamente de los gobiernos provinciales o municipales en un país federal. El Ministerio de Salud de la Nación, que prácticamente no cuenta con efectores directos ni autoridad de intervención en las decisiones de salud provinciales, ha tratado de aumentar su capacidad de rectoría a través de espacios de concertación interjurisdiccional y por el desarrollo de programas monitoreados en convenio con las provincias y los municipios, por los que provee recursos.

El eje central del desarrollo de la APS lo ha constituido la provisión gratuita de medicamentos esenciales en los centros de atención de primer nivel (CAPS Centros de Atención Primaria de la Salud), que se inició en 2002 con el programa REMEDIAR (ARGENTINA, 2006). Este programa, dirigido a población sin cobertura de obras sociales o seguros privados, también tenía entre sus objetivos modificar las pautas de utilización de servicios en un sistema tradicionalmente hospitalcéntrico, redireccionando la demanda de patologías ambulatorias hacia los CAPS.

A partir de 2004, en el marco del Plan Federal de Salud, otras acciones de fortalecimiento del primer nivel se iniciaron, entre ellas aquella destinada a formación de recursos humanos (médicos comunitarios) en articulación intersectorial con unidades académicas, y el financiamiento a los gobiernos provinciales para el fortalecimiento de redes de salud. Nos detendremos entre ellas en el Plan Nacer, creado en 2005, cuya finalidad es disminuir los índices de morbimortalidad materno-infantil, fortaleciendo la red pública de Atención Primaria en Salud. Brinda cobertura a embarazadas, puérperas hasta 45 días y niños/as menores de seis años sin otra cobertura, y funciona en los efectores de la red pública (CAPS y Hospitales), con población nominada y por acuerdo de nación con las provincias las que contratan los efectores. Las prestaciones de este plan, gratuitas para los usuarios, se encuentran nomencladas y el Estado paga por ellas. Esos recursos se destinan a la infraestructura, el equipamiento y la capacitación e incentivo de los recursos humanos (ARGENTINA, 200?). La incorporación a este programa es necesaria como condicionalidad para el cobro completo de la Asignación Universal por Hijo, económica por niño desde el embarazo que se ofrece a las familias que no cuentan con empleo o tienen empleo informal. Para recibir la totalidad del subsidio, los beneficiarios tienen que cumplir con el plan de vacunación completo y con chequeos anuales para conseguir el certificado de salud, y los chicos de hasta seis años deben inscribirse en el Plan Nacer. Dada la magnitud de la Asociación Universal por Hijo (AUH), debe ser remarcado su impacto en las situaciones sanitaria y educativa. Según datos del Ministerio de Salud de la Nación, durante los primeros diez meses del 2010 la inscripción al Plan Nacer creció más del 79\% respecto del mismo período del año anterior. En el plano educativo, la matrícula secundaria se incrementó un 20\%; en tanto que los controles de salud y la vacunación subieron un 56\% (ARGENTINA, 2011).

La Provincia de Buenos Aires, de la cual el municipio en estudio forma parte, participa de los programas nacionales REMEDIAR, NACER, Proyecto Funciones Esenciales de la Salud Pública (FESP) y Programa Federal de Salud (PROFE) (BUENOS AIRES, 200?).

Este sintético panorama nos permite afirmar que aunque el estudio realizado en un municipio no puede considerarse representativo del conjunto del país, 
habilita para ver la forma particular que adquieren las orientaciones generales y las potencialidades de transformación desde el primer nivel. En segundo lugar, fundamenta que en esta etapa se haya tomado como objeto de estudio al primer nivel de atención, aunque la definición de APS que forma parte de la investigaciónmarco sea la comprehensiva.

\section{El municipio en estudio}

El municipio de Lanús está ubicado en la Zona Sur de un complejo conglomerado urbano, el Gran Buenos Aires, compuesto por los 24 partidos que rodean a la Ciudad Autónoma de Buenos Aires. En él habitan 9.916.715 personas (INDEC, 2010), el 25\% de la población del país. Además de ser la mayor concentración urbana de la Argentina, es probablemente la de mayor complejidad social. Durante la crisis de hace una década el $64,4 \%$ de su población se encontraba bajo la línea de pobreza (INDEC, 2002) descendiendo en 2011 a $10 \%$ de pobreza y $3 \%$ de indigencia (INDEC, 2011).

El 38\% de sus habitantes no cuentan con cobertura de obra social o seguro privado de salud (INDEC, 2010). Los municipios que componen el Gran Buenos Aires forman parte de la Provincia de Buenos Aires, que organiza sus prestaciones en regiones sanitarias supramunicipales. En ellas hay prestadores provinciales y municipales, además de los privados y de obras sociales. Desde el 2006, el gobierno provincial implementa un seguro público de salud destinado a personas sin otra cobertura, con el objetivo de desarrollar acciones preventivas y brindar atención a través de los centros de salud municipales o efectores privados autorizados, a población nominada (BUENOS AIRES, 200?).

Habría que agregar que existe un intercambio y circulación permanente con la Ciudad de Buenos Aires, y los habitantes del Gran Buenos Aires incluyen en sus estrategias de cuidado de la salud el uso de servicios de salud de la Ciudad: el 32,5\% de los usuarios de consultas ambulatorias de los Hospitales Generales de Agudos de la Ciudad de Buenos Aires corresponden a habitantes del conurbano bonaerense (STOLKINER et al., 2003).

El Municipio de Lanús forma parte de la Región Sanitaria VIy tiene 459.263 habitantes (INDEC, 2010). Los servicios hospitalarios del subsector estatal son: un hospital interzonal general de agudos ("Evita"), un hospital zonal general de agudos ("Dr. Narciso López"), uno local general de agudos ("Dr. Arturo Melo") y uno zonal especializado en oncología, todos de dependencia provincial excepto el local que es de dependencia municipal (BUENOS AIRES, 200?). También es prestador del programa maternoinfantil del municipio un hospital pediátrico del sector privado (LANÚS, 2012a). La secretaría de salud municipal cuenta con 8 unidades sanitarias y 32 centros de atención de la salud, además de 1 Departamento de Medicina Preventiva que desarrolla ocho programas. Las unidades sanitarias se consideran parte del primer nivel de atención pero, a diferencia de los centros de salud, cuentan con servicio de guardia permanente en clínica médica, pediatría y enfermería y algunas de ellas realizan análisis clínicos, radiografías, colposcopias, Papanicolau y ecografías (LANÚS, 2012b), como visto en Figura 1.

Como ya se mencionara, en el primer nivel de atención confluyen recursos humanos y materiales y normativas provistos por los niveles nacional, provincial y municipal, aunque la responsabilidad de la gestión recaiga sobre el municipal. Entre los programas nacionales en ejecución en el municipio se cuentan el REMEDIAR, NACER y Médicos Comunitarios.

Como dato político a señalar, el municipio tuvo el mismo intendente o alcalde durante el período constitucional de 1973-1976 y desde el retorno a la democracia en 1983 hasta 2007, continuidad que probablemente estableció prácticas de gestión y redes de funcionamiento informal con potencial resistencia a cambios. La investigación se realizó durante la primera gestión posterior a ese período (la cual fue reelecta en 2011), que bien puede considerarse una etapa de transición.

\section{Metodología}

El objetivo de esta fase de la investigación fue comprender cómo los abordajes de la APS y los correspondientes modelos organizacionales de oferta y gestión de la APS son traducidos en servicios, prácticas y procesos, y cómo ocurre la interacción entre los actores involucrados en el territorio. Las herramientas aplicadas fueron: un cuestionario de Evaluación Rápida de APS, común a todos los países del estudio, al que se le hicieron algunas modificaciones de lenguaje para 
Figura 1. Mapa de efectores de Lanús, en 2011.

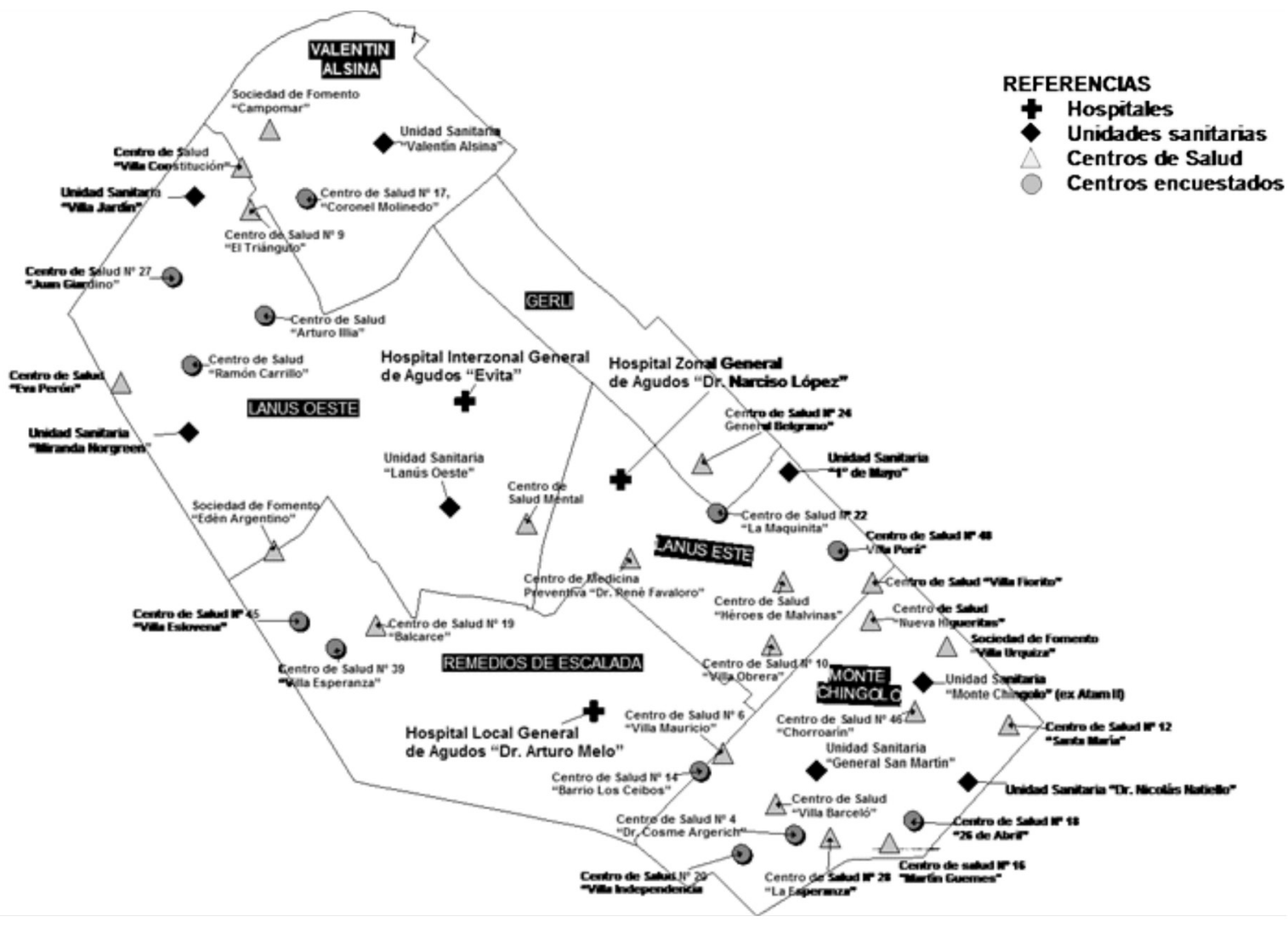

adaptarlo al español local; entrevistas semidirigidas a gestores del municipio, basadas en una guía de preguntas que abordaba las dimensiones del cuestionario, y un taller de devolución y debate de resultados al cual asistieron parte del personal de gestión de salud del municipio, los encuestadores (promotores y estudiantes) y docentes e investigadores del Departamento de Salud Comunitaria de la universidad.

El cuestionario, una adaptación del de Barbara Starfield realizada y validada por Almeida y Macinko (2006), fue aplicado a usuarios y profesionales de centros de salud. Se decidió dividir la categoría usuarios en adultos que consultan y adultos que llevan niños a la consulta, porque difería el nivel educativo entre ambos los grupos. Otra decisión metodológica fue aplicarlo solamente a los centros de salud y no a las unidades sanitarias, debido a la necesidad de trabajar unidades de análisis similares para la comparabilidad con los otros países, pese a la particularidad de este municipio de tener dos tipos de niveles de complejidad en el primer nivel de atención.

Para la aplicación del cuestionario, se realizó una muestra aleatoria de 12 centros de salud sobre los 32 existentes. En cada uno de ellos se encuestó a 20 usuarios seleccionados aleatoriamente por selección sistemática, y tres médicos. La muestra quedó conformada por 161 adultos consultantes, 79 adultos acompañantes y 36 médicos y fue aplicada por equipos constituidos por un estudiante de trabajo social de la Universidad y un promotor de salud del municipio, a los que se les proveyó una capacitación para la tarea.

Los resultados se analizaron según las ocho dimensiones para la evaluación del desempeño de la atención primaria del cuestionario: acceso, puerta de entrada, vínculo, portafolio de servicios, coordinación, enfoque familiar, orientación a la comunidad y formación 
profesional. Cada una de ellas está compuesta por una serie de variables, de cuyo promedio resulta el valor para cada dimensión, siendo seis el valor máximo y uno el mínimo. Finalmente, del promedio de las ocho dimensiones se obtiene el valor global de desempeño de la atención primaria.

\section{Resultados}

se expondrán los resultados por dimensión del cuestionario, triangulando con las observaciones que aparecieron en el taller de debate y en las entrevistas con gestores.

\section{Desempeño de la atención primaria en salud segundo la evaluación de profesionales y usuarios}

El índice global de desempeño de la APS en el municipio dio una puntuación mayor de cuatro, satisfactoria considerando que el valor máximo es seis. Hubo bastante coincidencia entre usuarios adultos $(4,01)$, acompañantes de niños $(4,27)$ y profesionales $(4,1)$. Como se destacará al analizar las dimensiones, los máximos valores los obtuvieron las variables de vínculo con los profesionales y formación profesional, y las puntuaciones más bajas correspondieron al acceso y a la orientación comunitaria (gráfico 1 ). Los resultados globales por dimensión son presentados en los gráfico 2, 3 y 4 .

La dimensión 'acceso' tuvo una valoración de poco más de tres sobre seis, baja en relación a otras. Dentro de ella las variables que más alto puntaje obtuvieron fueron la ausencia de aranceles, la facilidad para conseguir citas y la disponibilidad de medicamentos, teniendo esta última más alta valoración por parte de los acompañantes que de los usuarios adultos. El tiempo de espera tuvo una valoración intermedia, y fueron muy bajas las de cita telefónica y consulta luego de las 18 horas, o durante los fines de semana. Las respuestas de los profesionales fueron congruentes con las de los usuarios.

Gráfico 1. Resultados globales de las respuestas al cuestionario según dimensiones, Lanús, en 2011.

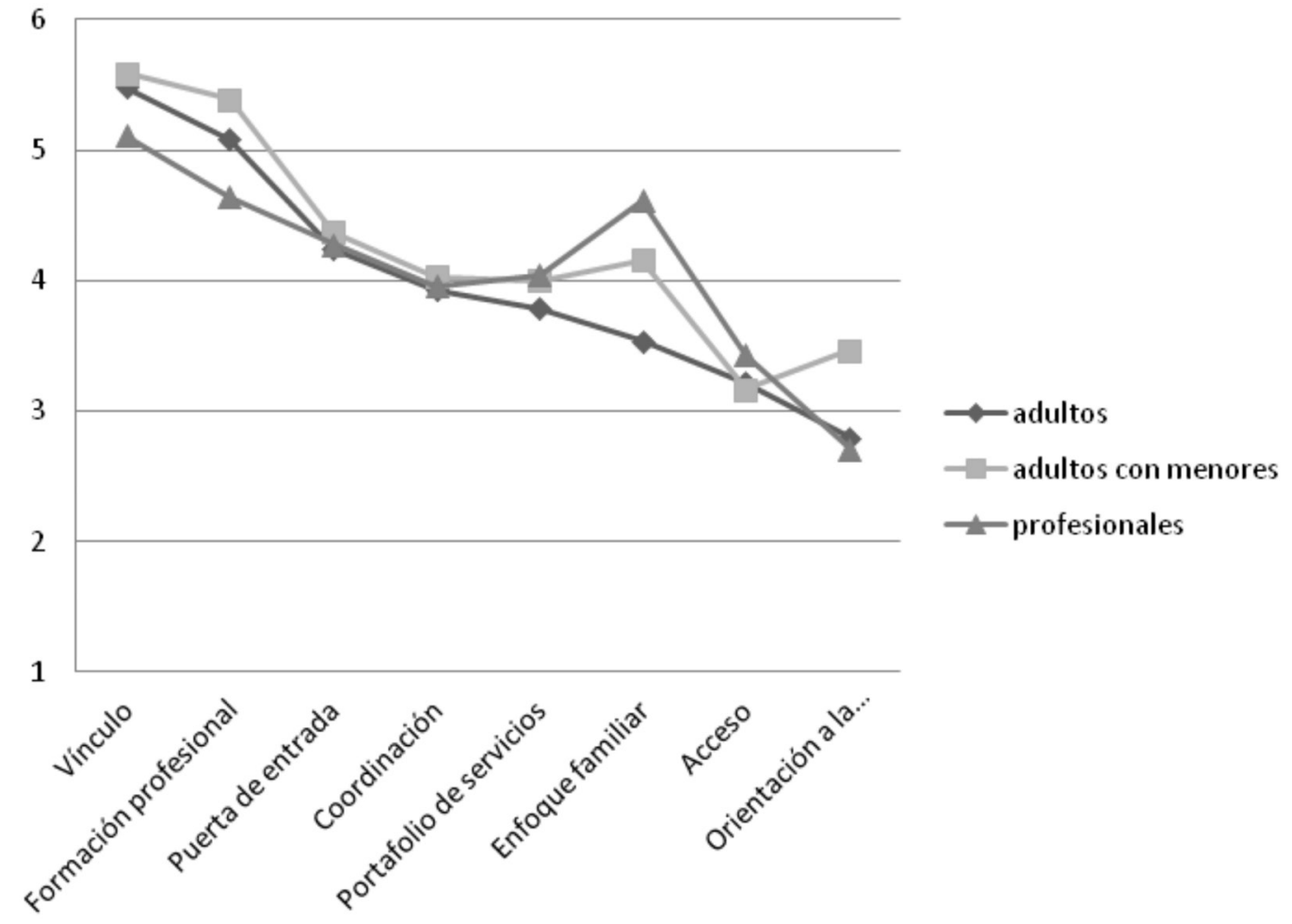


Gráfico 2. Resultados globales de las respuestas al cuestionario según dimensiones en usuarios adultos, Lanús, en 2011.

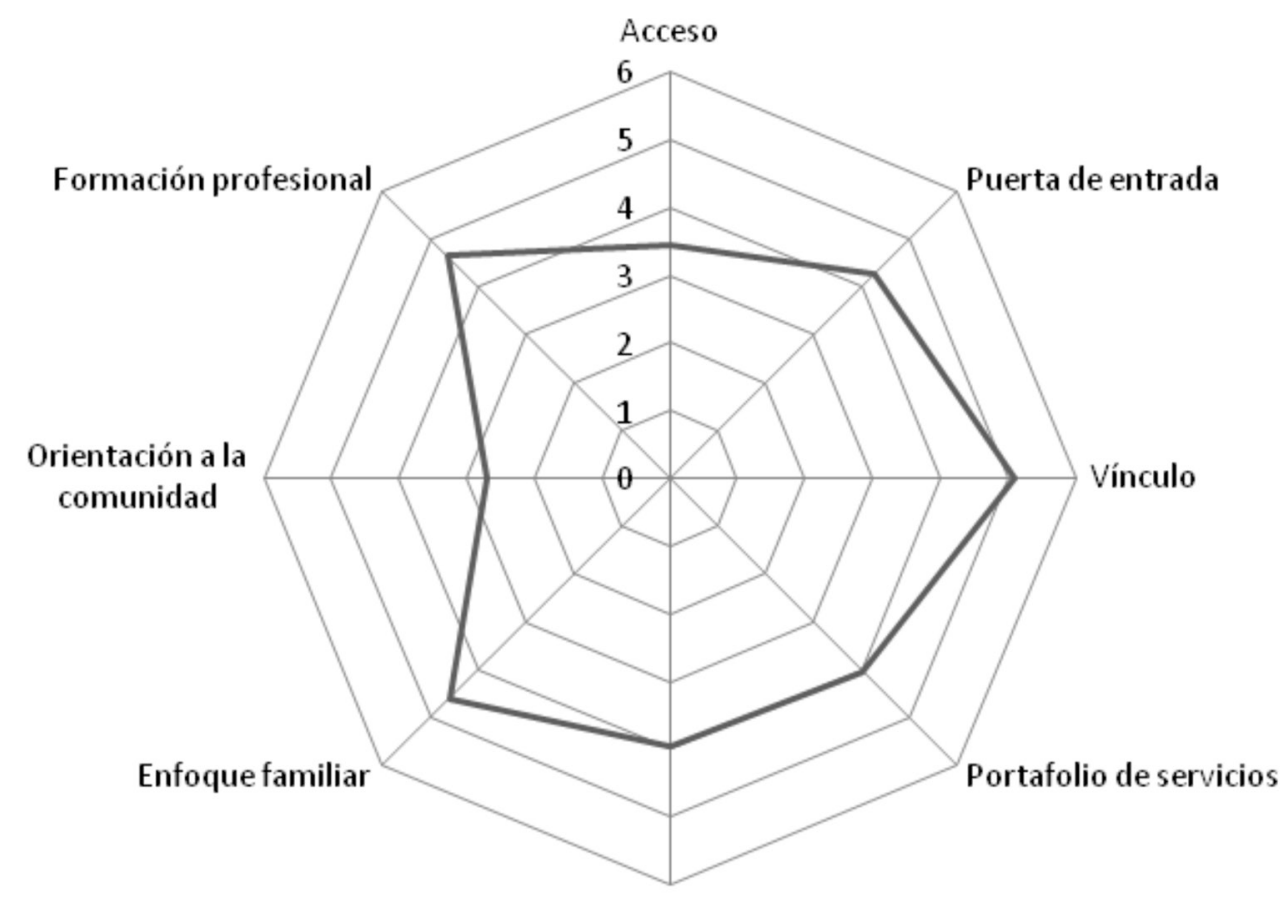

Fuente: Elaboración propia.

Coordinación

Gráfico 3. Resultados globales de las respuestas al cuestionario según dimensiones en usuarios acompañantes de niños, Lanús, en 2011.

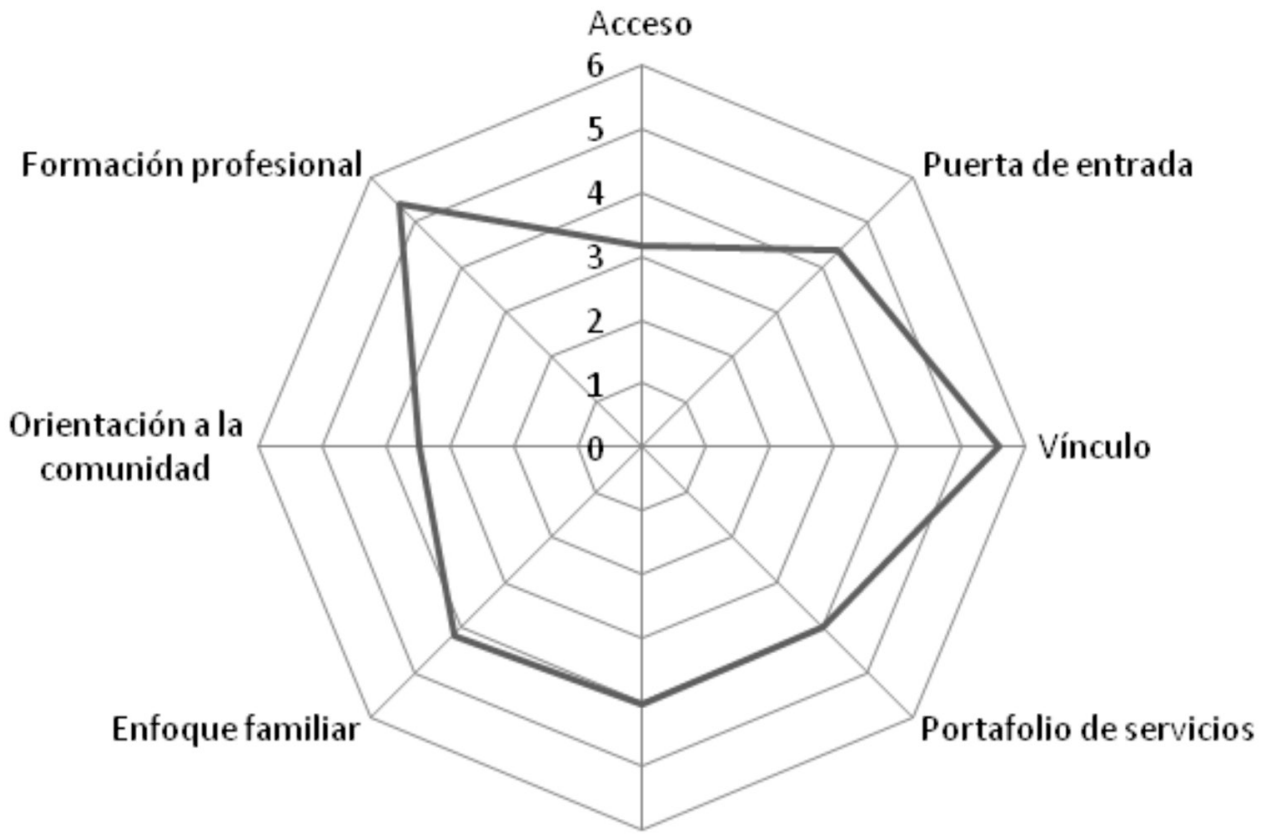

Fuente: Elaboración propia.

Coordinación 
Gráfico 4. Resultados globales de las respuestas al cuestionario según dimensiones en profesionales servicios, Lanús, en 2011.

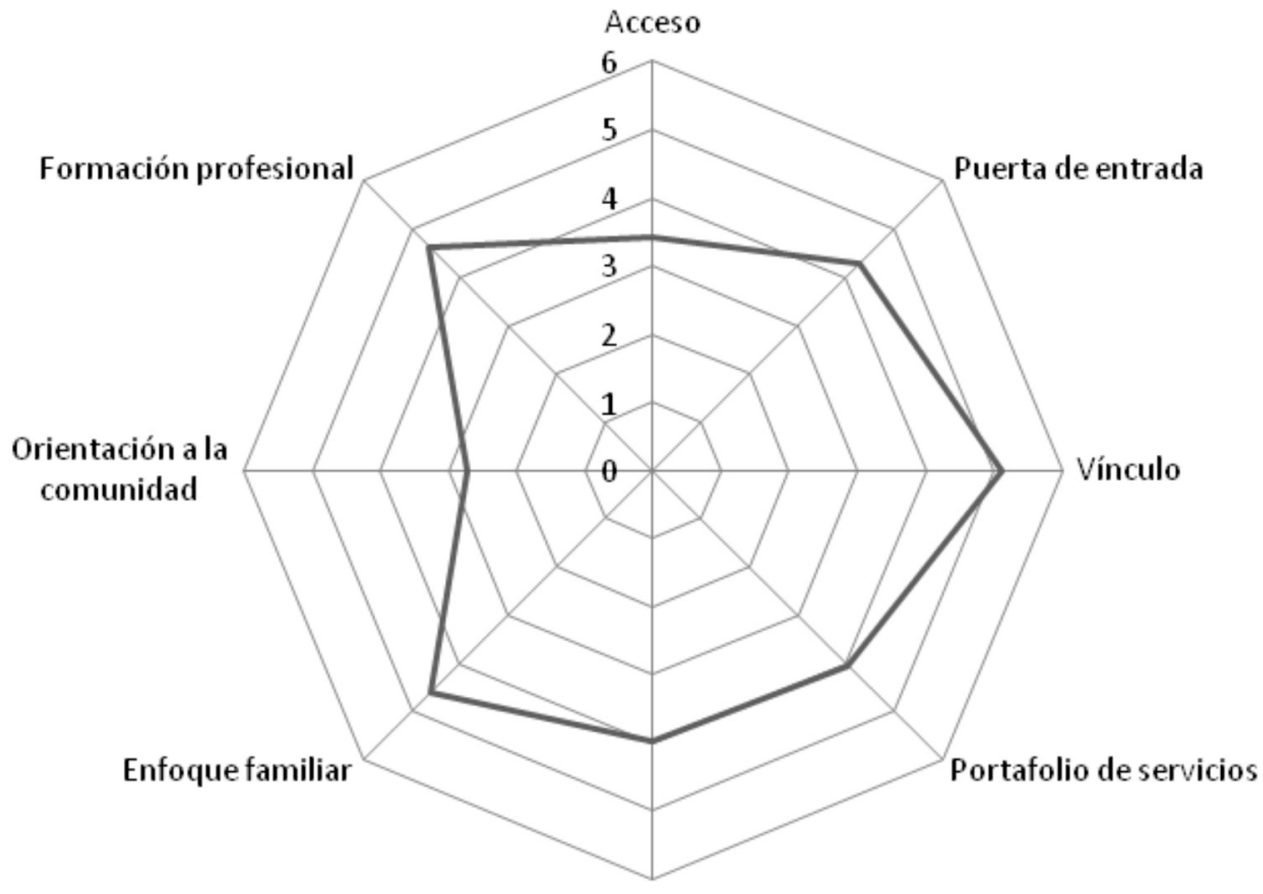

Coordinación

Fuente: Elaboración propia.

En el debate de resultados, los gestores observaron que se había producido un sesgo al no incluir en la muestra las unidades sanitarias que tienen guardia permanente y son el lugar de referencia para consulta fuera del horario de atención de los centros. Es necesario observar que el valor global de esta dimensión se logró al promediar algunos valores particularmente altos (ausencia de pago de aranceles y facilidad para acceder a la consulta) y otros notablemente bajos, debido a que el ítem evaluado (horario de atención posterior a las 18 horas, atención telefónica fuera de los horarios de funcionamiento, atención durante los fines de semana y otros) no está planeado en las prestaciones de estos establecimientos.

Para la dimensión 'puerta de entrada' en ambos los grupos de usuarios (adultos y acompañantes), la valoración fue alta para las variables de esta dimensión, difiriendo únicamente en que los acompañantes dan más respuestas positivas que los usuarios adultos ante la necesidad de consultar al centro de salud antes de ser dirigido a un especialista. Los profesionales también tuvieron una respuesta positiva alta a este ítem. La consulta por control preventivo tuvo un valor levemente más bajo que por aquella por problemas de salud, dentro de un rango alto para ambas.

La evaluación sobre el 'vínculo de los usuarios con el servicio' fue alta en ambos grupos de usuarios, situándose los valores entre cinco y seis para sus variables. Es posible sintetizar que los usuarios opinan que son atendidos usualmente por el mismo profesional, que éste les da un tiempo adecuado de consulta, anota los motivos, atiende a sus dudas y preguntas, responde de manera comprensible a ellas, conoce los medicamentos y, en menor medida, sabe si el usuario no puede conseguirlos.

Los profesionales dan un resultado similar en las preguntas comunes, dado que las hay específicas para ellos. Entre estas últimas, la variable que recibió la puntuación más baja es la que evalúa si tienen población adscripta. 
La dimensión 'cartera de servicios' contiene 19 variables referidas a distintos servicios de atención y prevención, que es esperable se brinden en un primer nivel de atención. Once de estas variables, en general las de atención más tradicional en este nivel, obtuvieron una valoración de cuatro o más puntos con bastante homogeneidad en las respuestas de usuarios adultos y acompañantes. Se situaron por debajo de los tres puntos la recolección de muestras para laboratorio (que no se realiza en los centros, sino en las unidades sanitarias), algunas actividades preventivas como consejería sobre consumo de alcohol y tabaquismo, actividad física y violencia familiar; la atención de patologías no graves en salud mental y la educación sobre accidentes domésticos. Hubo algunas discordancias con los profesionales dentro de las preguntas que son específicas para ellos, la existencia de protocolos para la actividad dio una puntuación intermedia.

En la dimensión 'coordinación' se incluyen variables importantes a los fines de esta investigación, que refieren a la continuidad de cuidados porque indaga sobre mecanismos de referencia y contrarreferencia. Lo primero a señalar es que muy pocos de los entrevistados responden haber realizado una consulta con un servicio especializado (en ambos los grupos el puntaje es menos de dos), no obstante es muy alta la puntuación en las respuestas de los profesionales a la pregunta sobre haber indicado consulta en otros niveles de atención. A su vez, dos valores que aparecen bajos en la encuesta a profesionales son los referentes a supervisión de necesidades de referencia y a la auditoría de registros de historia clínica. Como lo mencionamos, en el sistema de prestaciones del municipio, los hospitales son de dependencia provincial (excepto el Melo, que es de dependencia municipal) y, a su vez, es una práctica frecuente de la población realizar la consulta especializada en otras jurisdicciones o en los hospitales de la ciudad de Buenos Aires.

La dimensión 'enfoque familiar' obtuvo un valor global bajo en relación a otras, con algunas discordancias entre los grupos. Los profesionales reconocen la ausencia de una historia clínica familiar, pero afirman pedir información sobre enfermedades familiares, factores de riesgo social y también que pueden hablar con la familia del paciente. Los usuarios adultos consideran que es poco frecuente que se les pregunte sobre sus condiciones familiares o que los profesionales conozcan a su familia, sin embargo la puntuación de la variable que indaga sobre si los profesionales estarían dispuestos a hablar con su familia es alta. Finalmente, los acompañantes de consulta de niños difieren de los usuarios adultos, dando una valoración más alta al conocimiento que los profesionales tienen de la familia y a las preguntas sobre condiciones familiares. Esta última diferencia probablemente sea debido a las particularidades de los cuidados y enfoque pediátricos, necesariamente más referidos a la familia.

La dimensión 'orientación a la comunidad' resultó con una puntuación global baja. Aunque los resultados fueron bastante homogéneos entre los grupos, tuvo un valor global más alto en los acompañantes de niños. Esta diferencia parece deberse a la variable de 'oferta de servicios en las escuelas', que dio más alta en este grupo que en el de usuarios adultos y profesionales, quizás porque la mayoría de los acompañantes son padres o madres de niños en edad escolar y tienen otra percepción de estas actividades. También, los acompañantes dieron mayores respuestas afirmativas que los usuarios adultos frente a la pregunta si han sido consultados acerca de los servicios que se les brindan. Ambos grupos de usuarios coinciden en que no es frecuente la visita domiciliaria, y en que es baja la participación de la comunidad en los centros de salud y la relación de éstos con organizaciones de la sociedad civil. Las respuestas de los profesionales coinciden con las de los usuarios y, en sus variables específicas, registran que es baja la autonomía para reorganizar servicios y escasa la realización de encuestas para diagnosticar necesidades y problemas de la comunidad atendida.

Para la 'formación profesional', la valoración global de usuarios y acompañantes dio más alta que aquella de los profesionales, quizás porque estos últimos respondieron a preguntas específicas para ellos que modificaron el resultado global. Así, los usuarios y acompañantes opinaron que recomendarían este centro de salud a un familiar o amigo, que, en general, sus problemas son resueltos en el mismo, que usualmente hay por lo menos un médico/a y enfermero/a brindando atención y que el personal del centro se relaciona bien con la comunidad. Por su parte, los profesionales 
coincidieron con los usuarios en la valoración de las preguntas comunes, pero en las específicas ellos consideraron baja la capacitación en APS de médicos y profesionales, obteniendo una valoración satisfactoria en lo referente a adecuación del personal a las características de diversidad étnica y cultural de la población.

\section{Evaluación de gestores y taller de debate}

Las entrevistas con gestores fueron realizadas previamente a la aplicación del cuestionario y atendiendo a las dimensiones del mismo. Se entrevistó a tres gestores: secretario de salud del municipio, director del centro municipal de prevención y director de la división de establecimientos asistenciales. Sus respuestas corroboraron el cuestionario en casi todas las dimensiones, menos en enfoque familiar y comunitario, en los cuales su valoración parece ser más alta que la obtenida en los resultados de la encuesta. Señalaron que la consulta en los centros de salud se ha duplicado en los últimos tres años. Reconocieron que la provisión de medicamentos es amplia, basada en el Programa REMEDIAR y que la consulta es gratuita. En su opinión, la espera de los usuarios para ser atendido en los CAPS rara vez supera los 30 minutos y los usuarios son atendidos generalmente por el mismo profesional, pese a que no hay población adscrita. Coinciden en la necesidad de mejorar la supervisión de los profesionales/auditoría de historias clínicas. En la opinión de los gestores, la capacitación es ofrecida esencialmente por los programas verticales.

Los gestores reconocen que no hay barreras que impidan a un usuario acceder al sistema de salud en ninguno de sus niveles y reconocen las dificultades del sistema de referencia y contrarreferencia, considerando que sería mejor que hubiera niveles de atención más definidos para el acceso. Los gestores opinan que sería necesario un mayor presupuesto para la atención primaria y uno para el rápido mantenimiento de los centros de primer nivel.

En el taller de debate de resultados, una de sus observaciones fue el sesgo que en la dimensión de acceso había producido el no incluir las unidades sanitarias dentro de la muestra, lo cual habría influido fundamentalmente en los resultados. También plantearon, como problema, la existencia de centros de salud que no han surgido de la evaluación de necesidades, sino de acuerdos con dirigentes políticos barriales y cuya utilización es baja, pero que no pueden ser redistribuidos debido a que produciría alguna confrontación política. Manifestaron alguna discrepancia con respecto a los resultados de la dimensión de enfoque comunitario, comentando la existencia de un programa intersectorial con educación que lo promovería. El programa nacional, cuya presencia parece ser más percibida, es el REMEDIAR, pero son menos mencionados otros programas nacionales (por ejemplo el NACER) o provinciales (por ejemplo el Seguro Provincial de Salud), y pareciera ser necesaria una mejora en los mecanismos de articulación entre jurisdicciones (Nación-Provincia-Municipio).

Los resultados de la investigación les parecieron pertinentes como diagnóstico y útiles para el desarrollo de la gestión en términos de futuro.

\section{Discusión y conclusiones}

los resultados de esta fase del proyecto hicieron aparecer las particularidades que adquiere al nivel local el sistema de APS descripto en el estudio panorámico, así como especificidades del municipio en estudio y algunos interrogantes sobre la herramienta utilizada y las limitaciones de su aplicación.

En principio, se corroboró que la fragmentación y segmentación del sistema hace que la estrategia de APS dependa fundamentalmente del subsistema estatal y se identifique con el primer nivel de atención, a la par que se dificulta la continuidad de cuidados. El programa nacional, cuya presencia en el primer nivel se hizo más evidente, fue el de provisión gratuita de medicamentos, los otros aparecen menos mencionados o reconocidos aunque esto podría deberse que es en este nivel de atención donde tienden a confluir y alcanzar algún grado de integración, así como al hecho de que la herramienta no parece sensible a su detección.

El estudio mostró fortalezas y debilidades del primer nivel de atención en el municipio. Entre las primeras se cuentan el vínculo entre los usuarios y el personal de los centros de salud, entre las últimas el acceso, la falta de enfoque familiar y la escasa participación comunitaria. No obstante, algunos de los resultados (por ejemplo, en el acceso o la puerta de 
entrada) deben ser relativizados, teniendo en cuenta particularidades de la herramienta utilizada y decisiones metodológicas del estudio.

El bajo puntaje obtenido en la dimensión 'orientación a la comunidad' confluye con lo observado en la fase panorámica de la investigación respecto a la ausencia, en la implementación de la APS, de lineamientos o dispositivos institucionales que generen espacios para tal participación, por ejemplo, consejos locales de salud. También pueden relacionarse con esta dimensión algunos otros resultados, como la escasa aplicación de encuestas de necesidades o problemas y la falta de visitas domiciliarias, estos sugieren la presencia de una cultura institucional de concentración de la práctica en los efectores de salud, aún cuando 'el conocimiento de los problemas de salud de la comunidad' resultó una variable con buen puntaje.

El perfil obtenido por la encuesta da cuenta de un importante reconocimiento de los usuarios hacia el vínculo con los profesionales de los centros y su formación, el soporte fundamental de éste sería que generalmente son atendidos por el mismo profesional y que éste no tiene restricciones en cuanto al tiempo dedicado a la consulta. Teniendo en cuenta que, como se mostró en el presente estudio, no existe población adscripta y habría escasa auditoría de procedimientos e historias clínicas, esta pareciera ser una práctica desarrollada 'espontáneamente' por los agentes de salud, que debiera ser promovida y resguardada. De todos modos, sería interesante triangular estos resultados con un estudio cualitativo que permitiera indagar cómo se construye y en qué representaciones se sostiene este reconocimiento. Se trataría de problematizar el 'encuentro' entre población y agentes de salud, teniendo en cuenta los componentes socioculturales del mismo (LUPPI et al., 1993). Es de señalar también como hipótesis que este vínculo de los usuarios con los profesionales haya sido un factor que incidió en la diferencia entre los puntajes entre usuarios y profesionales en algunas de las dimensiones, en donde los puntajes de los usuarios fueron más altos que los otorgados por los profesionales.

Por otro lado, la herramienta utilizada permite un perfil minucioso de las prácticas del primer nivel de atención y resulta útil para la comparación. En la revisión bibliográfica se encontraron investigaciones en las que se la utiliza para comparar servicios privados y estatales (VEGA; MARTÍNEZ; ACOSTA, 2009). Pero presenta limitaciones que fueron motivo de debate en el equipo de investigación. Una limitación es que se asigna el mismo valor de ponderación a variables que no son equivalentes en cuanto a su peso en la utilización de los servicios. Un ejemplo sería la baja puntuación que obtuvo la dimensión de acceso. Como se expuso, se llegó a este resultado por una combinación de valores extremos altos y bajos. Sin embargo, tratándose de población de bajos recursos económicos, la accesibilidad económica facilitada por la ausencia de aranceles y la gratuidad de los medicamentos (ambas variables con puntajes altos) deben tener un peso significativamente mayor en el acceso a los servicios que, por ejemplo, la posibilidad de solicitar turno de manera telefónica (el puntaje más bajo). En estudios realizados durante los 1990, con población pobre de otro municipio, la imposibilidad de comprar los medicamentos y el cobro de aranceles aparecían como las principales causas de desestimación de la consulta aún en caso de enfermedad (BARCALA; STOLKINER, 2000). Finalmente, con respecto a la dimensión de acceso, dado que se entrevistan usuarios que 'utilizan' los centros, no se puede deducir de ello conclusiones con respecto a la población en general, lo que requeriría una encuesta poblacional aleatoria.

Como otro ejemplo, uno de los problemas que los gestores consideraron relevante, el que hace a continuidad de cuidados, sólo se indaga en cinco preguntas sobre sistemas de referencia y contrarreferencia - en la dimensión de coordinación - siendo uno de los nudos de gestión más complicados probablemente. A esto se suma la limitación de las preguntas para indagar integralidad en la atención o intersectorialidad, y son pocas las preguntas sobre atención de enfermedades crónicas y salud mental.

Cabe también mencionar que el resultado de la dimensión 'puerta de entrada' resulta relativizado por aplicarse a usuarios de los centros de primer nivel, es posible que personas de ese mismo grupo poblacional asistan directamente a los hospitales locales o de otras jurisdicciones, sin pasar por el primer nivel. Esto fue seńalado por los participantes del taller y de los gestores. Queda también abierta una pregunta sobre la 
discrepancia existente entre la respuesta de los profesionales acerca de la frecuencia con que derivan a especialistas y la escasa consulta con especialistas, reportada por los usuarios encuestados. Esta discrepancia abriría líneas de indagación y preguntas específicas.

Entre las decisiones metodológicas a considerar en el análisis de los resultados, una es el hecho que los promotores de salud, que formaban parte del equipo encuestador, pertenecen a la comunidad y conocen los centros de salud, lo cual pudo haber influido en las respuestas de los usuarios y de los médicos, así como también que las entrevistas fueran todas realizadas antes de la consulta, un hecho que podría modificar las apreciaciones de los usuarios. La segunda, ya mencionada, es que la selección muestral se realizó sobre centros de salud y no incluyó unidades sanitarias, esto influyó en los resultados, por lo menos, de la variable acceso dado que la atención permanente ( 24 horas) se realiza en las unidades, así como también la toma de muestras.

Teniendo en cuenta estas observaciones, pareciera que el cuestionario podría ser un primer paso para detectar problemas, para luego trabajar una estrategia de triangulación de métodos (MINAYO et al., 2005) que facilitara un nivel mayor de comprensión de las particularidades local.

La interacción del equipo de investigación con los gestores y actores del municipio, que mostraron colaboración y apertura al debate de resultados, fue otro de los resultados del proyecto. El proceso de indagación posibilitó sinergias entre los investigadores y los actores del municipio que participaron del mismo. El objetivo de que los conocimientos generados por la investigación se convirtiesen en un insumo para la gestión, la discusión e intercambio en torno a los resultados de la investigación fueron parte del mismo proceso de trabajo. Prueba de esto es que el cambio de Secretario de Salud Municipal ocurrido promediando la segunda mitad de la implementación de esta fase no obstaculizó la continuidad del trabajo, dando cuenta de la fortaleza de la relación establecida.

En este sentido, la posibilidad de que los distintos aspectos señalados por la investigación sean tenidos en cuenta en el próximo plan de salud, como lo han manifestado las autoridades municipales, constituye uno de los efectos más potentes del estudio.

\section{Agradecimientos}

Agradecemos a los autores de la versión original del cuestionario, Celia Almeida (FIOCRUZ) y James Macinko (New York University) por autorizar la aplicación y mínima adaptación del mismo para el presente estudio.

\section{Referencias}

ALAZRAQUI, M.; SPINELLI, H. Desigualdades en salud en el nivel local/municipal. Buenos Aires: Universidad Nacional de Lanús, 2008. 239p.

ALMEIDA, C. Delimitación del campo de la investigación en sistemas y servicios de salud: desarrollo histórico y tendencias. Cuadernos para Discusión, Rio de Janeiro, n. 1, 2000, p. 11-35.

ALMEIDA, C.; MACINKO, J. Desenvolvimento de Sistemas e Serviços de Saúde. Validaçao de uma metodología rápida das características organizacionais e do desempenho dos serviços de atenção básica do Sistema Único de Saúde (SUS) em nível local. Brasília: OPAS, 2006. 215p.

ARGENTINA. Ministerio de Salud de la Nación. PROAPS. REMEDIAR. Caracterización de los centros de salud del País. 2006. Disponível em: <http://www.remediar.gov.ar>. Acesso em: 2 out. 2008.
Argentina salud. Época II, año 2, n. 7, jan./2011, p. 18-20.

Remediar+Redes Hoy. [200?]. Disponível em: <http:// www.remediar.gob.ar/>. Acesso em: 2 fev. 2012.

Plan Nacer. [200?]. Disponível em: <http://www.ms.gba. gov.ar/PlanNacer/documentacion/brochure.pdf>. Acesso em: 10 fev. 2012.

BARCALA, A.; STOLKINER, A. Accesibilidad a Servicios de Salud de familias con necesidades básicas insatisfechas (NBI): Estudio de Caso VIII Anuario de Investigaciones. Buenos Aires: Facultad de Psicología de la Universidad de Buenos Aires, 2000, p. 282-294.

BELMARTINO, S. Una década de reforma de la atención médica en la Argentina. Revista Salud Colectiva, v. 1, n. 2, p. 155-171, mai./ago., 2005. 
BUENOS AIRES (Província). Ministerio de Salud. ProducciónProgramas Nacionales. [200?a]. Disponível em: <http://www. ms.gba.gov.ar/programas/programasnacionales.html>. Acesso em: 9 dez. 2011.

Seguro Público de Salud. [200?b]. Disponível em: <http:// www.ms.gba.gov.ar/programas/seguropublico/Introduccion. html>. Acesso em: 5 fev. 2012.

Región Sanitaria VI. 2012. Disponível em: <http://www. ms.gba.gov.ar/regiones/RSVI/index.html>. Acesso em: 5 fev. 2012.

BURSZTYN, I. et al. Notas para el Estudio de Atención Primaria en Sistemas de Salud Segmentados. Revista de Salud Pública, Colombia, v. 12, Supplemento 1, abr, 2010, p. 77-85.

INSTITUTO NACIONAL DE ESTADÍSTICAS Y CENSOS (INDEC). Censo 2010. Disponível em: <http://www.censo2010.indec.gov. ar/resultadosdefinitivos.asp>. Acesso em: 3 fev. 2012.

Incidencia de la Pobreza e Indigencia en el Gran Buenos Aires, 2003. Disponível em: <http://www.indec.gov.ar/nuevaweb/ cuadros/74/incid_07_03.pdf>. Acesso em: 3 fev. 2012.

Encuesta Permanente de Hogares Incidencia de la Pobreza y de la Indigencia Resultados del primer semestre 2011. 2011. Disponível em: <http://www.indec.mecon.gov.ar/nuevaweb/ cuadros/74/pob_tot_1sem11.pdf>. Acesso em: 3 fev. 2012.

Censo 2010, Cuadro P12-P. Provincia de Buenos Aires, 24 partidos del Gran Buenos Aires. Población en viviendas particulares por tipo de cobertura desalud, según sexoy grupo deedad. 2010.Disponível em: $\quad$ <http://www.censo2010.indec.gov.ar/CuadrosDefinitivos/ P12-P_buenos_aires_gba.pdf>. Acesso em 3 fev. 2012.

Censo 2010, Cuadro P2-D. Provincia de Buenos Aires, partido Lanús. Población total por sexo e índice de masculinidad, según edad en años simples y grupos quinquenales de edad. 2010. Disponível em: <http://www.censo2010.indec.gov.ar/CuadrosDefinitivos/ P2-D_6_434.pdf>. Acesso em: 11 dez. 2011.
LUPPI, I. et al. Investigaciones evaluativas en servicios de salud. Cuadernos Médico Sociales, Rosario, v. 64, jul. 1993, p. 31-54.

MINAYO, M.C.S.; GONÇALVES DE ASSIS, S.; RAMOS DE SOUZA, E. (Org). Evaluación por triangulación de Métodos-Abordaje de programas sociales. Buenos Aires: Lugar Editorial, 2005. p. 71-104.

RED DE INVESTIGACIÓN EN SISTEMAS Y SERVICIOS DE SALUD EN EL CONO SUR. Estudio multicéntrico en atención primaria en salud: modelos asistenciales, integración al sistema de salud e intersectorialidad en contextos urbanos en Argentina, Brasil, Paraguay y Uruguay. 2011. Disponível em: <http://www.ensp. fiocruz.br/parcerias/redsalud>. Acesso em: 12 dez. 2011.

STOLKINER, A.; COMES, Y.; GARBUS, P. Alcances y potencialidades de la Atención Primaria de la Salud en Argentina. Ciência \& Saúde Coletiva, v. 16, n. 6, 2011, p. 2807-2816.

STOLKINER, A. et al. Descentralización y Equidad en Salud: Estudio sobre la utilización de servicios de salud de la Ciudad de Buenos Aires por parte de usuarios pobres del conurbano bonaerense. Serie Seminarios Salud y Políticas Públicas, CEDES, Buenos Aires, 2003, p. 3.

UNIVERSIDAD NACIONAL DE LANÚS. Estatutos. 2010. Disponível em: <http://www.unla.edu.ar/documentos/Estatuto_ Universidad_Nacional_de_Lanus_03-03-2010.pdf>. Acesso em: 10 dez. 2010.

VEGA, R.; MARTÍNEZ, J.; ACOSTA, N. Evaluación rápida del desempeño de la red pública de servicios de salud de Suba en el logro de los atributos de la Atención Primaria de Salud-APS. Revista Gerencia y Políticas de Salud, v. 8, n. 16, 2009, p. 165-190.

Recebido para publicação em março de 2012.

Versão definitiva em agosto de 2012.

Suporte financiero: International Development Research Centre (IDRC), Canadá.

Conflicto de intereses: Inexistente. 